\title{
Novel Flexible Self-Powered Humidity Detection enabled by Triboelectric Effect
}

\author{
Si Wang ${ }^{1}$, Huiling Tai*, ${ }^{*}$, Guangzhong Xie ${ }^{1}$, Yuanjie Su${ }^{1}$ Yadong Jiang ${ }^{1}$ \\ 1 School of Optoelectronic Information, University of Electronic Science and Technology of China \\ (UESTC), Chengdu 610054, China \\ *To whom correspondence should be addressed: taitai1980@uestc.edu.cn
}

\begin{abstract}
In this work, a fundamental new approach for flexible self-powered humidity detection was proposed. By integrating humidity-sensitive materials into the nanogenerator (TENG), the degree of ambient moisture can be actively detected at room temperature, where the output voltage of the TENG has a reversely proportional relationship with relative humidity. The morphology and chemical properties of the fabricated sensor based on $\mathrm{Ce}-\mathrm{ZnO}$ heterostructure nanoparticles were characterized by SEM, FTIR and UV-visible spectroscopy respectively. In addition, the prepared flexible self-powered triboelectric humidity sensor (FTHS) holds high humidity response of $\sim 70 \%$, fast response/recovery time of $23 \mathrm{~s}$ and $24 \mathrm{~s}$ (@90\% respectively) and stable repeatability. Finally, the underlying working principle of the proposed sensor was discussed in detail. This research not only provides a simple, cost-effective, and robust technique in self-powered humidity sensing but also successfully realizes the integration of humidity-sensitive materials in the TENG to achieve integrated compatibility in humidity detection.
\end{abstract}

Key words: humidity sensor, self-powered, triboelectrification, flexible, Ce-ZnO

\section{Introduction}

Humidity monitoring and control play a crucial role in quality of manufacture, food safety and comfortability of daily life. By far, various approaches have been developed and applied to enhance the accuracy and sensitivity of humidity sensors [1-2]. However, all of these conventional sensing techniques require external power source for the sustainable operation, hence, it is significant to bring the self-powered technology to humidity detection. In recent years, triboelectric nanogenerator (TENG) has been developed to harvest a varieties of ambient mechanical energies [3-4], which could be a new paradigm for large scale energy. In this work, a fundamental new approach for flexible self-powered humidity detection was proposed. By integrating humidity-sensitive materials $(\mathrm{Ce}-\mathrm{ZnO})$ into the nanogenerator (TENG), the degree of ambient moisture can be actively detected at room temperature because of the good humidity sensing properties of the prepared FTHS.

\section{Experimental section}

The preparation process of the FTHS was schematically illustrated in Fig. 1(a). The flexible PET substrates coated with $\mathrm{Cu}$ electrodes were selected to support the humidity sensitive film $(\mathrm{Ce}-\mathrm{ZnO})$ and the frictional film (PDMS film modified by template method). The Ce-ZnO nanoparticles were synthetized by hydrothermal synthesis method and then formed the sensitive film by spray method. Finally, the FTHS was motivated by a linear motor (JZK-10, Sinoceramics, Inc) with a tunable impact speed, force and frequency. The output voltage of the FTHS was measured by Keithley 6514 system electrometer.

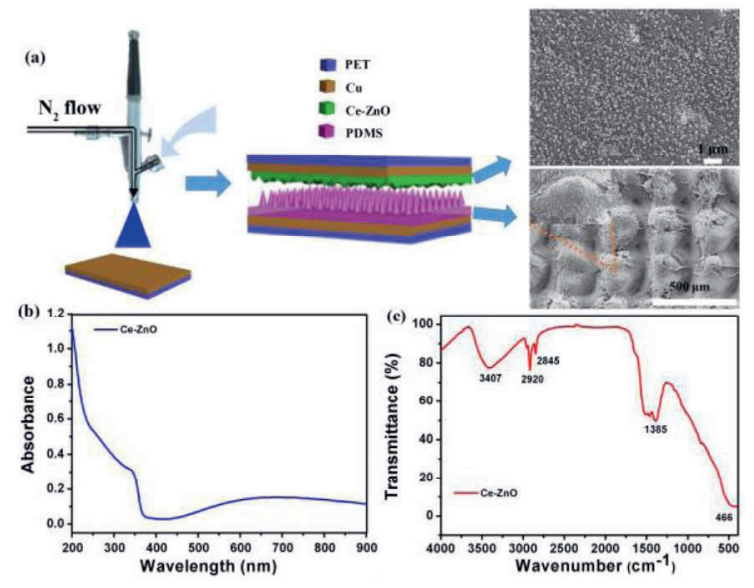

Fig. 1. (a) Schematic diagram of the preparation of the FTHS, and the SEM images of Ce-ZnO heterostructure nanoparticles and PDMS film, respectively; (b) UV-visible spectroscopy and (c) FTIR spectra of prepared humidity sensitive films. 


\section{Results and discussion}

The SEM images, FTIR spectra and UV-visible spectroscopy of the prepared film are shown in Fig. 1. To explore the humidity sensing properties, a measurement setup is built up as sketched in Fig. 2(a). The FTHS was exposed to different relative humidity environments through various saturated saline solution from $11.3 \% \mathrm{RH}$ to $97.5 \% \mathrm{RH}$. Fig. 2(b) and (c) show the output performance of the prepared FTHS triggered by impact with different driving distances from $54-56.4 \mathrm{~mm}$ at the fixed frequency of $1 \mathrm{~Hz}$. With the driving distance increasing, the sensitive film are closer to the friction film, resulting in an increase of the output signal, and $56 \mathrm{~mm}$ was selected in the following test. The output voltage of FTHS monotonously drops down with increasing the relative humidity (Fig. 3(a)), then, the average output voltage was extracted from Fig. 3(a) and plotted in Fig. 3(b) as a function of $\mathrm{RH}$, where the Ce-ZnO film exhibits a good slope together with linearity $\left(R^{2}\right)$ of 0.9043 . Fig. $3(c)$ is the dynamic response of the prepared FTHS upon exposure to $97.3 \% \mathrm{RH}$, where the response and recovery time are defined as the sensor achieves $90 \%$ of total voltage variation. And a fast response and recovery time of $23 \mathrm{~s}$ and 24 $\mathrm{s}$ was observed, respectively, which confirms that FTHS is a rapid and effective technique for humidity detection. Fig. 3(d) demonstrates the excellent repeatability of the FTHS for a very little response fluctuation during repeated desiccating and moistening cycles when tested at the dry air and $97.3 \% \mathrm{RH}$ alternately for four cycles. Owing to high specific surface area of $\mathrm{Ce}$ doped $\mathrm{ZnO}$ and its good adsorption on water molecules, we can realize self-powered test of environment humidity by detecting the voltage changes of the FTHS.
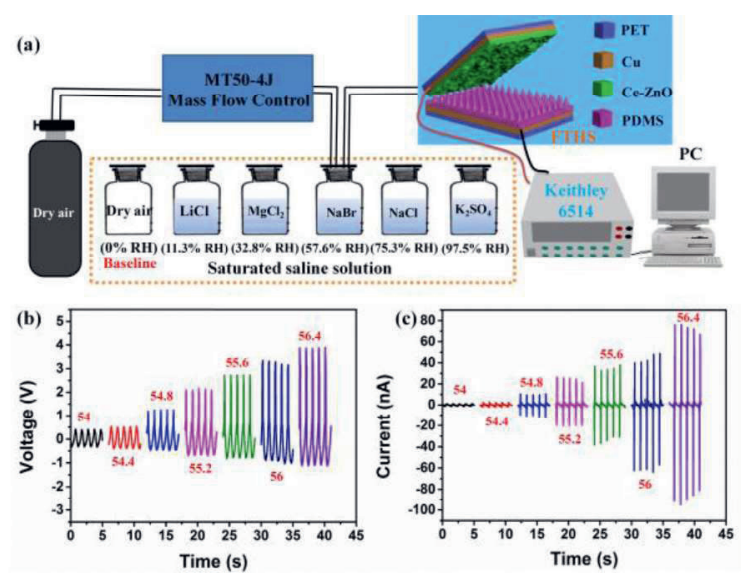

Fig. 2. (a) Experimental setup for humidity-sensing properties measurement of the as-fabricated selfpowered humidity sensor; the influence of driving distance on (b) the open circuit voltage and (c) short circuit current of the FTHS from 54-56.4 $\mathrm{mm}$ at the fixed frequency of $1 \mathrm{~Hz}$.
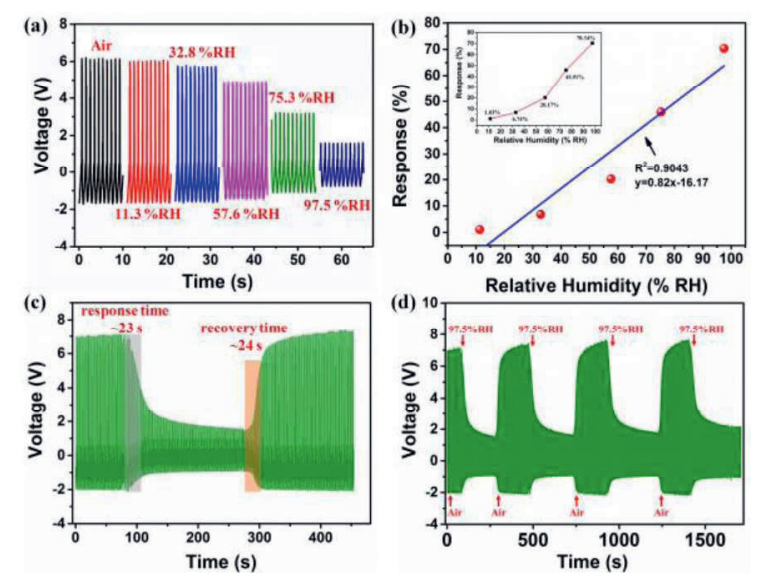

Fig. 3. (a) The output voltage of the FTHS under various relative humidity $(R H)$ from $11.3 \% R H$ to $97.5 \% \mathrm{RH}$; (b) response-RH fitting curve of the prepared sensor. Insert: the response curve of the FTHS; (c) the dynamic response of the FTHS; (d) the repeatability of fabricated sensor with four cycles ranging from dry air to $97.5 \% \mathrm{RH}$.

\section{Conclusions}

In summary, the Ce-ZnO nanoparticles were integrated into the TENG to achieve the selfpowered humidity detection. In addition, the prepared FTHS holds high humidity response of $\sim 70 \%$, fast response/recovery time of $23 \mathrm{~s}$ and $24 \mathrm{~s}$ and stable repeatability. This research provides a cost-effective way to broaden the applicability of TENG in the detection of humidity.

\section{Acknowledgments}

This work is partially supported by the Funds for Creative Research Groups of China (NO. 61421002), the National Natural Science Foundation of China (Grant Nos. 61571097, 61604033) and the National Postdoctoral Program for Innovative Talents (Grant No. BX201600026).

\section{References}

[1] V. K. Tomer, S. Duhan, Highly sensitive and stable relative humidity sensors based on WO3 modified mesoporous silica, Appl. Phys. Lett. 106, 802- 808 (2015); doi: 10.1063/1.4908116

[2] J. Xie, H. Wang, Highly sensitive humidity sensor based on quartz crystal microbalance coated with ZnO colloid spheres, Sens. Actuators, B: Chem. 177, 1083-1088 (2013); doi: 10.1016/j.snb.2012.12.033

[3] W. Q. Yang, J. Chen, Harvesting Energy from the Natural Vibration of Human Walking. ACS Nano 7, 11317-11324 (2013); doi:10.1021/nn405175z

[4] W. Q. Yang, J. Chen, 3D stack integrated triboelectric nanogenerator for harvesting vibration energy. Adv. Funct. Mater. 24, (2014) 4090-4096; doi: 10.1002/adfm.201304211 